\title{
Platelet Activation and Chemokine Release Are Related to Local Neutrophil-Dominant Inflammation During Hyperacute Human Stroke
}

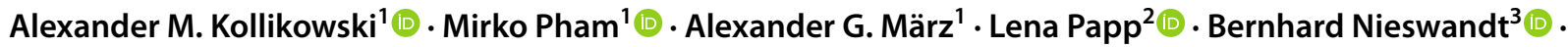 \\ Guido Stoll ${ }^{2}$. Michael K. Schuhmann ${ }^{2} \mathbb{0}$
}

Received: 22 April 2021 / Revised: 15 July 2021 / Accepted: 4 August 2021 / Published online: 28 August 2021

(c) The Author(s) 2021

\begin{abstract}
Experimental evidence has emerged that local platelet activation contributes to inflammation and infarct formation in acute ischemic stroke (AIS) which awaits confirmation in human studies. We conducted a prospective observational study on 258 consecutive patients undergoing mechanical thrombectomy (MT) due to large-vessel-occlusion stroke of the anterior circulation (08/2018-05/2020). Intraprocedural microcatheter aspiration of $1 \mathrm{ml}$ of local (occlusion condition) and systemic arterial blood samples (self-control) was performed according to a prespecified protocol. The samples were analyzed for differential leukocyte counts, platelet counts, and plasma levels of the platelet-derived neutrophil-activating chemokine C-X-C-motif ligand (CXCL) 4 (PF-4), the neutrophil attractant CXCL7 (NAP-2), and myeloperoxidase (MPO). The clinical-biological relevance of these variables was corroborated by specific associations with molecular-cellular, structural-radiological, hemodynamic, and clinical-functional parameters. Seventy consecutive patients fulfilling all predefined criteria entered analysis. Mean local CXCL4 (+ 39\%: $571 \mathrm{vs} 410 \mathrm{ng} / \mathrm{ml}, P=.0095)$ and CXCL7 (+ 9\%: $693 \mathrm{vs} 636 \mathrm{ng} / \mathrm{ml}, P=.013$ ) concentrations were higher compared with self-controls. Local platelet counts were lower $(-10 \%: 347,582$ vs 383,284/ $\mu$ l, $P=.0052)$, whereas neutrophil counts were elevated $(+10 \%$ : 6022 vs 5485/ $\mu 1, P=0.0027)$. Correlation analyses revealed associations between local platelet and neutrophil counts $(r=0.27, P=.034)$, and between CXCL7 and MPO $(r=0.24, P=.048)$. Local CXCL4 was associated with the angiographic degree of reperfusion following recanalization $(r=-0.2523, P=.0479)$. Functional outcome at discharge correlated with local MPO concentrations $(r=0.3832, P=.0014)$ and platelet counts $(r=0.288$, $P=.0181)$. This study provides human evidence of cerebral platelet activation and platelet-neutrophil interactions during AIS and points to the relevance of per-ischemic thrombo-inflammatory mechanisms to impaired reperfusion and worse functional outcome following recanalization.
\end{abstract}

Keywords CXCL4 $\cdot$ PF $4 \cdot$ CXCL7 $\cdot$ NAP-2 $\cdot$ Ischemic stroke $\cdot$ Chemokines

\section{Introduction}

Mirko Pham

pham_m@ukw.de

$\triangle$ Michael K. Schuhmann schuhmann_m@ukw.de

1 Department of Neuroradiology, University Hospital of Würzburg, Josef-Schneider-Straße 11, 97080 Würzburg, Germany

2 Department of Neurology, University Hospital of Würzburg, Josef-Schneider-Straße 11, 97080 Würzburg, Germany

3 Institute of Experimental Biomedicine, University Hospital and Rudolf Virchow Center, University of Würzburg, Würzburg, Germany
The pathobiology of acute ischemic stroke (AIS) encompasses a plethora of intertwined processes within the ischemic territory such as energy failure, excitotoxicity, anoxic depolarization, apoptosis, and, more recently recognized, neuroinflammation [1,2]. Evidence in rodents and baboons points to a prominent role of platelets and coagulation factors which accumulate early at sites of cerebral infarction [3, 4], and drive inflammation-related tissue damage [5]. Human evidence supporting the causative "thromboinflammatory" interplay between thrombotic and inflammatory mechanisms within the compromised ischemic vascular compartment is not available [1], but would have profound implications regarding the relevance and adequate timing of 
conceptually sound add-on treatments to use before and/or after recanalization therapy for large-vessel-occlusion stroke [6-8]. A major limitation in stroke research is that human samples are restricted mainly to peripheral venous blood or lumbar cerebrospinal fluid (CSF) that are both obtained far remote from and frequently with time delay to the ischemic event [9]. Recently, we and other investigators established the method of endovascular microcatheter aspiration of cerebral-ischemic blood from within the center of the occluded arterial compartment during human AIS [10, 11]. Using this novel approach which reduces interference by effects other than local $[10,11]$, it was now possible to quantify the cerebral concentrations of the platelet-derived neutrophilactivating chemokine CXC motif ligand (CXCL) 4 (platelet factor 4, PF-4), and the neutrophil attractant CXCL7 (neutrophil-activating peptide 2, NAP-2). These chemokines represent highly abundant releasates upon platelet activation $[12,13]$, and are considered both (1) as biomarkers of high platelet activity in the systemic circulation[14] and (2) as crucial regulators of neutrophil function $[13,15]$. The clinical-biological relevance of our findings was addressed by analyzing specific associations with leukocellular, structural-radiological, and hemodynamic-functional parameters assessed during ischemia, and short-term functional outcome following recanalization.

\section{Methods}

This prospective single-center cross-sectional study (08/2018-05/2020) was approved by the ethics committee of the University of Würzburg (\#135/17) and complies with the Declaration of Helsinki. All patients or their legal representatives provided written informed consent. From August 2018 to May 2020, microcatheter aspiration of cerebral arterial blood samples during acute large-vessel-occlusion stroke of the anterior circulation was attempted in 258 consecutive patients undergoing mechanical thrombectomy (MT). Briefly, $1 \mathrm{ml}$ of citrate-phosphate-dextrose-adenine (CPDA1)-anticoagulated local blood from within the cerebralischemic arterial compartment and systemic control samples from the cervical internal carotid artery (ICA) were obtained according to the protocol published previously by our group [10]. As defined per protocol, the human occlusion condition was precisely matched with the most widely practiced middle cerebral artery occlusion (MCAO) models of acute focal ischemic stroke [16]. Details on the inclusion criteria are provided in the Supplemental Information. Blood sampling was followed by sample processing and analysis including differential leukocyte counts, platelet counts, and plasma preparation [10]. After centrifugation to obtain cell-free samples, plasma was immediately stored at $-20{ }^{\circ} \mathrm{C}$. Plasma levels of CXCL4/7 and myeloperoxidase (MPO) were quantified by commercially available kits according to the manufacturers' specifications: enzyme-linked immune sorbent assay of CXCL4-Thermo scientific [Cat.Nr.: EHPF4], CXCL7-abcam [Cat.Nr.: ab100613]; flow cytometric quantification of MPO-Biolegend [Cat.Nr.: 740561]. The extension of cerebral infarction prior to recanalization was measured through the Alberta Stroke Program Early CT Score (ASPECTS; CT: Somatom Definition AS; Siemens Healthineers, Erlangen, Germany) [17]. Post-processing algorithms (syngo iFLOW software; Siemens Healthineers, Erlangen, Germany) were applied for parametric color coding of digital subtraction angiography (DSA; angiography system: Siemens Artis Q, Siemens Healthineers, Erlangen, Germany) series (4 frames per second) and for the quantification of collateralization by means of retrograde collateral flow as assessed by collateral transit time (relative time to peak opacification, rTTP) [18]. rTTP was determined by using a region-of-interest (ROI) analysis, where predetermined ROI locations reflected the site of cerebral sampling and the vascular target region typical of receiving retrograde collateral supply under occlusion condition. rTTP was calculated by subtracting the time to peak opacification (TTP) of ROI 1 (circular, placed at the lacerum segment of the internal carotid artery, measuring $10.80 \mathrm{~mm}^{2}$ ) from the TTP of ROI 2 (circular, placed centered at the mid-insular sampling location, measuring $309.60 \mathrm{~mm}^{2}$ ). The angiographic degree of final reperfusion following recanalization therapy was graded by the modified treatment in cerebral infarction scale (mTICI). Functional clinical outcome was assessed by means of the modified Rankin Scale (mRS) at hospital discharge.

The manuscript was prepared according to the STROBE (Strengthening the Reporting of Observational Studies in Epidemiology) statement for observational studies [19].

\section{Statistical Analysis}

Statistical analyses were performed using GraphPad Prism (GraphPad Prism 9.0.1, GraphPad Software, San Diego, CA, USA). Gaussian distribution was tested by the D'Agostino and Pearson omnibus normality test. The Wilcoxon signed-rank test was used for comparison of related samples. Spearman's rank correlation coefficient was applied to determine principal associations between local cerebral-ischemic and clinical-radiological target variables to establish the relevance to human AIS. Data are given as mean with $95 \%$ confidence interval (CI), as median with interquartile range [IQR], or as absolute and relative frequency distribution, unless otherwise specified. All $P$-values reported are 2 -sided with $P<0.05$ being considered statistically significant. 


\section{Results}

The entire eligible cohort comprised $n=364$ consecutive patients who were treated by MT between August 2018 and May 2020. $N=39$ patients were excluded for posterior circulation occlusion and $n=21$ patients for bilateral or multifocal occlusion location. $N=46$ patients did not qualify for inclusion upon invasive angiographic imaging either due to spontaneous recanalization or sub-occlusion with residual antegrade flow before MT. Microcatheter aspiration of ischemic blood samples was attempted in $n=258$ patients with angiographically proven large-vessel-occlusion of the following target sites: ICA-T, middle cerebral artery M1, and proximal M2 segment. Aspiration of cerebral blood samples succeeded in $n=143$ patients (55\%). Out of these, $n=70$ consecutive patients (27\%) met all a priori defined sampling, interventional, and laboratory criteria of inclusion, and entered data analyses [10]. The full patient flow without missing cases is given in the Supplementary Information. All clinical, radiologic, interventional, and sampling-related patient characteristics are summarized in Table 1.

Results of cell and platelet counts, chemokines and clinical correlation analyses are presented in Fig. 1 and the Supplementary Information. Local cerebral-ischemic plasma levels of CXCL4 (+39\%: $571 \mathrm{ng} / \mathrm{ml}, 95 \% \mathrm{CI}=420$ to $722 \mathrm{vs} 410 \mathrm{ng} / \mathrm{ml}, 95 \% \mathrm{CI}=298$ to $522, P=0.0095)$ and CXCL7 (+ 9\%: $693 \mathrm{ng} / \mathrm{ml}, 95 \% \mathrm{CI}=549$ to 838 vs $636 \mathrm{ng} /$ $\mathrm{ml}, 95 \% \mathrm{CI}=492$ to $781, P=0.013)$ were higher as compared to intraindividual systemic control samples (Fig. 1a). Concomitantly, cerebral-ischemic platelet counts were reduced by $10 \%(347,582 / \mu 1,95 \% \mathrm{CI}=298,711$ to 396,453 vs $383,284 / \mu 1,95 \% \mathrm{CI}=333,194$ to $433,374, P=0.0052$ ), whereas neutrophil counts were elevated by $+10 \%(6022 /$ $\mu \mathrm{l}, 95 \% \mathrm{CI}=5188$ to 6855 vs $5485 / \mu \mathrm{l}, 95 \% \mathrm{CI}=4799$ to $6171, P=0.0027$; Fig. 1a). No significant differences were observed for lymphocyte or monocyte counts, and MPO (Supplementary Information). Subgroup analyses according to whether or not intravenous thrombolysis was performed did not reveal differences with regard to local CXCL4 (656 ng/ml, 95\% CI = 401 to 910 vs $506 \mathrm{ng} / \mathrm{ml}$, $95 \% \mathrm{CI}=316$ to $696, P=0.3315)$, CXCL7 $(766 \mathrm{ng} / \mathrm{ml}$, $95 \% \mathrm{CI}=545$ to 988 vs $636 \mathrm{ng} / \mathrm{ml}, 95 \% \mathrm{CI}=448$ to 828 , $P=0.2785)$, and MPO (45 ng/ml, 95\% CI $=32$ to $57 \mathrm{vs}$ $47 \mathrm{ng} / \mathrm{ml}, 95 \% \mathrm{CI}=32$ to $62, P=0.8462)$ concentrations.

Local cerebral-ischemic platelet counts were correlated with CXCL4 ( $r=0.2583, P=0.0463$; Fig. 1b). Neither of the cell counts correlated with CXCL7. Direct correlations were found between local platelet and neutrophil counts ( $r=0.2652, P=0.0342)$ as well as local CXCL7 and MPO concentrations ( $r=0.2446, P=0.0478$; Fig. $1 \mathrm{~b})$, with MPO being only associated with neutrophil counts $(r=0.3257$,
Table 1 Main clinical, radiologic, interventional, and samplingrelated patient characteristics

\begin{tabular}{|c|c|}
\hline Demographics & $n=70$ \\
\hline Age, y & $78[67-83]$ \\
\hline Male & $25(35)$ \\
\hline \multicolumn{2}{|l|}{ Medical history } \\
\hline Hypertension & $61(87)$ \\
\hline Diabetes mellitus & $14(20)$ \\
\hline Hyperlipidemia & $23(33)$ \\
\hline Atrial fibrillation & $40(57)$ \\
\hline Smoking history & $16(23)$ \\
\hline \multicolumn{2}{|l|}{ Baseline medication } \\
\hline Anti-thrombotic medication & $34(49)$ \\
\hline Antihypertensive drugs & $58(83)$ \\
\hline \multicolumn{2}{|l|}{ Clinical characteristics } \\
\hline Systolic blood pressure, $\mathrm{mmHg}$ & 159 [149-180] \\
\hline Diastolic blood pressure, $\mathrm{mmHg}$ & 86 [71-96] \\
\hline Heart rate, $\min ^{-1}$ & 80 [70-97] \\
\hline NIHSS at presentation & $15[10-18]$ \\
\hline Unknown time of symptom onset & $18(26)$ \\
\hline ASPECTS at presentation & 8 [7-9] \\
\hline \multicolumn{2}{|l|}{ Treatment } \\
\hline \multicolumn{2}{|l|}{ Thrombolysis } \\
\hline IV rt-PA & $29(41)$ \\
\hline \multicolumn{2}{|l|}{ Intervention } \\
\hline Onset-to-puncture, $\min$ & 245 [169-336] \\
\hline \multicolumn{2}{|l|}{ Angiographic occlusion location ${ }^{a}$} \\
\hline M1 & $48(68)$ \\
\hline M2 & $20(29)$ \\
\hline ICA & $12(17)$ \\
\hline rTTP, sec & $3[2-4]$ \\
\hline Stent-retrieval maneuvers & $2[1-3]$ \\
\hline Successful recanalization $^{\mathrm{b}}$ & $61(87)$ \\
\hline Duration of MT procedure, min & $69[50-114]$ \\
\hline Onset-to-final-recanalization time, $\min$ & 323 [247-379] \\
\hline \multicolumn{2}{|l|}{ Sampling } \\
\hline Onset-to-distal sampling time, min & 278 [204-360] \\
\hline Onset-to-carotid sampling time, $\min$ & 334 [262-401] \\
\hline \multicolumn{2}{|l|}{ Outcome } \\
\hline $\mathrm{mRS}$ at discharge & $3[1-5]$ \\
\hline Death during hospital stay & $10(14)$ \\
\hline
\end{tabular}

Data are given as median [interquartile range] for continuous variables, and number (percentage) for categorial variables

NIHSS, National Institutes of Health Stroke Scale; ASPECTS, Alberta Stroke Program Early CT score; $I V r t-P A$, intravenous recombinant tissue plasminogen activator; $M 1 / M 2$, middle cerebral artery section; $I C A$, internal carotid artery; $r T T P$, relative time to peak opacification; $M T$, mechanical thrombectomy; $m R S$, modified Rankin Scale

${ }^{a}$ Including multiple sites per patient

${ }^{\mathrm{b}}$ Defined as mTICI (modified treatment in cerebral infarction) scale $2 \mathrm{~b}$ or 3 


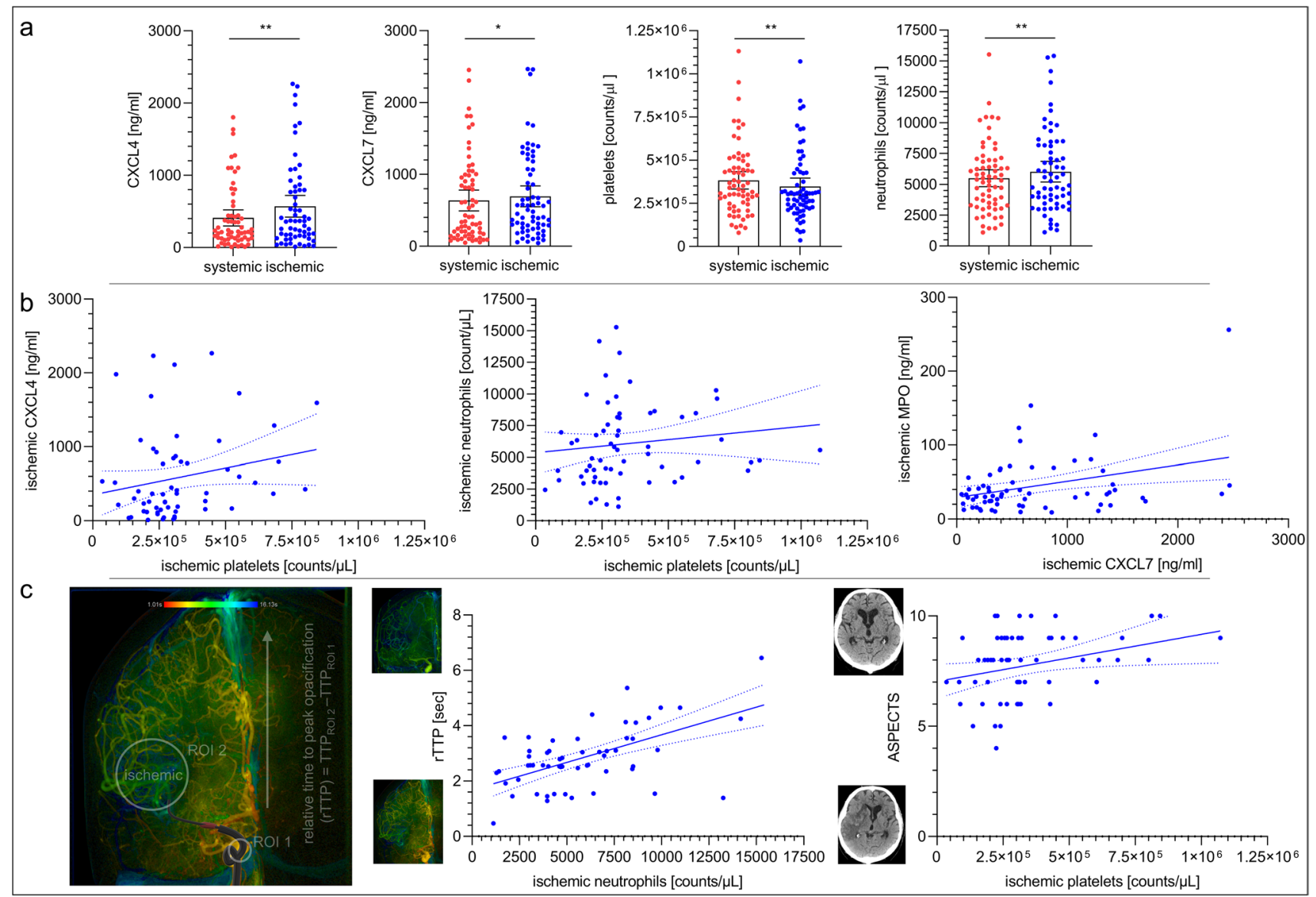

Fig. 1 Cerebral platelet activation is paralleled by neutrophil recruitment during acute ischemic stroke. a Quantification of plasmatic CXCL4 (PF-4)/CXCL7 (NAP-2) concentrations $(n=62 / n=67)$ as well as platelet $(n=67)$ and neutrophil $(n=65)$ counts. Each dot represents related systemic versus cerebral-ischemic blood samples during acute human stroke. Scatter dot plot with mean and 95\% confidence interval (CI). Wilcoxon matched-pairs signed-rank test. $* P<.05 ; * * P<.005$. b Left panel: Linear correlation between ischemic CXCL4 concentrations ( $y$-axis) and ischemic platelet counts ( $x$-axis). Spearman rank order correlation $(n=60), r=0.2583$, $P=.0463$. Middle panel: Linear correlation between ischemic neutrophil counts ( $y$-axis) and ischemic platelet counts ( $x$-axis). Spearman rank order correlation $(n=64), r=0.2652, P=.0342$. Right panel: Linear correlation between ischemic MPO ( $y$-axis) and CXCL7 $(x$-axis) concentrations. Spearman rank order correlation $(n=66)$, $r=0.2446, P=.0478$. Each dot represents a single case with correlation line. Short-dotted lines: $95 \%$ CI of the correlation line. c Left panel: Color-coded digital subtraction angiography (DSA) visualizing collateral transit time (relative time to peak opacification, rTTP) and local ischemic blood sampling (target region: ischemic, white circle) during embolic occlusion (red) of the M1 segment of the middle cerebral artery, micro- (distal to embolic lesion), and intermediate catheter (proximal to embolic lesion) in place (black). Middle panel: Linear correlation between collateral transit time (rTTP; $y$-axis, including representative images of good and poor collateral status) and ischemic neutrophil counts ( $x$-axis). Spearman rank order correlation $(n=57), r=0.4733, P=.0002$. Right panel: Linear correlation between radiological infarct extension under occlusion (Alberta Stroke Program Early CT score, ASPECTS; $y$-axis, including representative images of progressive and absent infarction) and ischemic platelet counts ( $x$-axis). Spearman rank order correlation $(n=62)$, $r=0.3075, P=.0151$. Each dot represents a single case with correlation line Short-dotted lines: 95\% CI of the correlation line.

of the MT procedure $(r=0.2526, P=0.0495)$. Functional clinical outcome (mRS) was correlated with local MPO concentrations ( $r=0.3832, P=0.0014)$ and platelet counts $(r=0.288, P=0.0181)$. All other results are given in the Supplementary Information. 


\section{Discussion}

In this study, we provide evidence for platelet activation and platelet-neutrophil interactions within the collateral circulation of AIS patients as reflected by the local increase of the platelet-derived chemokines CXCL4 and CXCL7 [15]. These observations were accompanied by locally reduced platelet counts and elevated neutrophil counts indicating that platelet activation within the ischemic cerebral vasculature is paralleled by acute local neutrophil-dominant inflammation.

Platelets are the most abundant and most rapidly available source of CXCL4 and CXCL7 which, following platelet activation, are found in thousand-fold higher concentrations than present in normal plasma [15]. While CXCL4 is almost exclusively stored in platelet $\alpha$-granules [12], CXCL7 is also expressed in peripheral blood mononuclear cells (PBMC), however only in minute amounts [20]. CXCL7 promotes directed intravascular recruitment of neutrophils[21] and induces the degranulation of neutrophil primary and secondary granules [15], the former of which contain large amounts of MPO as one of their major protein constituents [22]. CXCL4, on the other hand, can induce firm adhesion of neutrophils to endothelial cells, while exerting no chemotactic activity [23]. Unlike the plasmatic markers of platelet activation, MPO concentrations did not significantly differ between the sampling sites. This finding is not contradictory to local neutrophil activation, but in line with the interpretation that cerebral ischemia, i.e., a local cerebral reduction in oxygen partial pressure [24], may significantly alter the pattern of neutrophil metabolism [25]. Therefore, the association between CXCL7 and MPO concentrations is highly suggestive for local neutrophil activation and/or modulation by platelets [15]. Lower numbers of platelets, probably caused by attachment to the ischemic endothelium, consumption, and/or platelet necrosis, were associated with more advanced stroke demarcation at presentation $[4,26]$. Interestingly, local CXCL4 concentrations were correlated with the angiographic degree of final reperfusion following recanalization therapy and the duration of the intervention, which might reflect both platelet-leukocyte interactions downstream the site of the (macro)vascular embolic lesion and clot interaction with the endothelium at the site of the embolic lesion [23, 27, 28]. Furthermore, the magnitude of cerebral neutrophil influx was associated with impairment of collateral flow [28]. Conceivably, neutrophil influx may lead to collateral failure-associated cerebral infarction, possibly by increasing vascular resistance due to augmented blood viscosity[29] and/or by cellular obstruction [30].

A limitation of the current study is its observational design and use of soluble cell activation markers. In addition, no adjustment for multiple testing was made which can be justified by the exploratory nature of this study. As strength, we provide comparative, direct, and integrative human cerebral data obtained during stroke emergency care which support the pathophysiological concept of stroke-induced "thromboinflammation."[1] Thereby, we shift the experimental focus from thrombo-inflammatory mechanisms during the phase of reperfusion to clinical pertinence already during the earliest phases of stroke formation under occlusive condition [5, 6].

In summary, we report on local cerebral platelet activation and potential platelet-neutrophil interactions within the compromised ischemic cerebral vasculature of large-vesselocclusion stroke patients and point to the mutual significance of these cells for acute tissue damage. This should prompt further experimental and clinical investigations into local cerebral neutrophil-platelet interactions under occlusion condition that elucidate whether promising anti-thrombotic and/or anti-inflammatory treatments must be administered well before the initiation of recanalization therapies to exert maximum protective effects [8].

Supplementary Information The online version contains supplementary material available at https://doi.org/10.1007/s12975-021-00938-w.

Author Contribution AMK, MP, GS, and MKS designed the study; AMK was responsible for patient inclusion; AMK, MP, and AGM acquired data and biospecimen; LP and MKS performed laboratory analyses; AMK and MKS analyzed the data; AMK, MP, AGM, LP, $\mathrm{BN}, \mathrm{GS}$, and MKS interpreted the data; AMK and MKS supervised the study; AMK, MP, GS, and MKS wrote the manuscript; and all authors read and approved the final manuscript.

Funding Open Access funding enabled and organized by Projekt DEAL. This study was funded by the Deutsche Forschungsgemeinschaft (DFG) project 374031971-TRR240 (MP, BN, GS, and MKS; Gerok position held by AMK). AMK was supported by the DFG project 413657723 (Clinician Scientist programme UNION CVD).

Data Availability The datasets generated during and/or analyzed during the current study are available from the corresponding author on reasonable request.

Code Availability Not applicable.

\section{Declarations}

Ethics Approval Approval was obtained from the ethics committee of the University of Würzburg (\#135/17). The procedures used in this study adhere to the tenets of the Declaration of Helsinki.

Consent to Participate. Informed consent was obtained from all individuals included in the study or from their legal representatives.

Consent for Publication. The authors affirm that all individuals or their legal representatives provided informed consent for publication.

Conflict of Interest The authors declare no conflicts of interest.

Open Access This article is licensed under a Creative Commons Attribution 4.0 International License, which permits use, sharing, 
adaptation, distribution and reproduction in any medium or format, as long as you give appropriate credit to the original author(s) and the source, provide a link to the Creative Commons licence, and indicate if changes were made. The images or other third party material in this article are included in the article's Creative Commons licence, unless indicated otherwise in a credit line to the material. If material is not included in the article's Creative Commons licence and your intended use is not permitted by statutory regulation or exceeds the permitted use, you will need to obtain permission directly from the copyright holder. To view a copy of this licence, visit http://creativecommons. org/licenses/by/4.0/.

\section{References}

1. Stoll G, Nieswandt B. Thrombo-inflammation in acute ischaemic stroke - implications for treatment. Nat Rev Neurol. 2019;15:473-81.

2. Chamorro Á, Dirnagl U, Urra X, Planas AM. Neuroprotection in acute stroke: targeting excitotoxicity, oxidative and nitrosative stress, and inflammation. Lancet Neurol. 2016;15:869-81.

3. Okada Y, Copeland BR, Fitridge R, Koziol JA, del Zoppo GJ. Fibrin contributes to microvascular obstructions and parenchymal changes during early focal cerebral ischemia and reperfusion. Stroke. 1994;25:1847-53 (discussion 1853-4).

4. Del Zoppo GJ, Copeland BR, Harker LA, Waltz TA, Zyroff J, Hanson SR, et al. Experimental acute thrombotic stroke in baboons. Stroke. 1986;17:1254-65.

5. Schuhmann MK, Bieber M, Franke M, Kollikowski AM, Stegner $\mathrm{D}$, Heinze KG, et al. Platelets and lymphocytes drive progressive penumbral tissue loss during middle cerebral artery occlusion in mice. J Neuroinflammation. 2021;18:46.

6. Stoll G, Pham M. Beyond recanalization - a call for action in acute stroke. Nat Rev Neurol. 2020;16:591-2.

7. Dirnagl U. Thomas Willis Lecture. Stroke. 2016;47:2148-53.

8. Kollikowski AM, Cattus F, Haag J, Feick J, März AG, Weidner F, et al. Progression of cerebral infarction before and after thrombectomy is modified by prehospital pathways. J Neurointerv Surg. 2021;neurintsurg-2020-017155.

9. Allan SM, Tyrrell PJ, Rothwell NJ. Interleukin-1 and neuronal injury. Nat Rev Immunol. 2005;5:629-40.

10. Kollikowski AM, Schuhmann MK, Nieswandt B, Müllges W, Stoll G, Pham M. Local leukocyte invasion during hyperacute human ischemic stroke. Ann Neurol. 2020;87:466-79.

11. Fraser JF, Collier LA, Gorman AA, Martha SR, Salmeron KE, Trout AL, et al. The Blood And Clot Thrombectomy Registry And Collaboration (BACTRAC) protocol: novel method for evaluating human stroke. J Neurointerv Surg. 2019;11:265-70.

12. Deppermann C, Cherpokova D, Nurden P, Schulz J, Thielmann I, Kraft P, et al. Gray platelet syndrome and defective thrombo-inflammation in Nbeal2-deficient mice. J Clin Invest. 2013;123:3331-42.

13. Brandt E, Petersen F, Ludwig A, Ehlert JE, Bock L, Flad HD. The beta-thromboglobulins and platelet factor 4: blood platelet-derived CXC chemokines with divergent roles in early neutrophil regulation. J Leukoc Biol. 2000;67:471-8.

14. Snoep JD, Roest M, Barendrecht AD, De Groot PG, Rosendaal FR, Van Der Bom JG. High platelet reactivity is associated with myocardial infarction in premenopausal women: a populationbased case-control study. J Thromb Haemost. 2010;8:906-13.

15. Brandt E, Ludwig A, Petersen F, Flad HD. Platelet-derived CXC chemokines: old players in new games. Immunol Rev. 2000;177:204-16.
16. Sommer CJ. Ischemic stroke: experimental models and reality. Acta Neuropathol. 2017;133:245-61.

17. Barber PA, Demchuk AM, Zhang J, Buchan AM. Validity and reliability of a quantitative computed tomography score in predicting outcome of hyperacute stroke before thrombolytic therapy. ASPECTS Study Group. Alberta Stroke Programme Early CT Score. Lancet. 2000;355:1670-4.

18. Gölitz P, Muehlen I, Gerner ST, Knossalla F, Doerfler A. Ultraearly assessed reperfusion status after middle cerebral artery recanalization predicting clinical outcome. Acta Neurol Scand. 2018;137:609-17.

19. von Elm E, Altman DG, Egger M, Pocock SJ, Gøtzsche PC, Vandenbroucke JP, et al. The Strengthening the Reporting of Observational Studies in Epidemiology (STROBE) Statement: guidelines for reporting observational studies. Int J Surg. 2014;12:1495-9.

20. Smith C, Damås JK, Otterdal K, Øie E, Sandberg WJ, Yndestad A, et al. Increased levels of neutrophil-activating peptide- 2 in acute coronary syndromes: possible role of platelet-mediated vascular inflammation. J Am Coll Cardiol. 2006;48:1591-9.

21. Ghasemzadeh M, Kaplan ZS, Alwis I, Schoenwaelder SM, Ashworth KJ, Westein E, et al. The CXCR1/2 ligand NAP-2 promotes directed intravascular leukocyte migration through platelet thrombi. Blood. 2013;121:4555-66.

22. Borregaard N, Cowland JB. Granules of the human neutrophilic polymorphonuclear leukocyte. Blood. 1997;89:3503-21.

23. Kasper B, Brandt E, Ernst M, Petersen F. Neutrophil adhesion to endothelial cells induced by platelet factor 4 requires sequential activation of Ras, Syk, and JNK MAP kinases. Blood. 2006;107:1768-75.

24. Martha SR, Collier LA, Davis SM, Erol A, Lukins D, Pennypacker KR, et al. Evaluation of sex differences in acid/base and electrolyte concentrations in acute large vessel stroke. Exp Neurol. 2020;323:113078.

25. Davis WB, Husney RM, Wewers MD, Herzyk DJ, Sagone AL. Effect of $\mathrm{O} 2$ partial pressure on the myeloperoxidase pathway of neutrophils. J Appl Physiol. 1988;65:1995-2003.

26. Denorme F, Manne BK, Portier I, Eustes AS, Kosaka Y, Kile BT, et al. Platelet necrosis mediates ischemic stroke outcome in mice. Blood. 2020;135:429-40.

27. Desilles J-P, Syvannarath V, Di Meglio L, Ducroux C, Boisseau W, Louedec L, et al. (2018) Downstream microvascular thrombosis in cortical venules is an early response to proximal cerebral arterial occlusion. J Am Heart Assoc. 2018;7:e007804.

28. del Zoppo GJ, Schmid-Schönbein GW, Mori E, Copeland BR, Chang CM. Polymorphonuclear leukocytes occlude capillaries following middle cerebral artery occlusion and reperfusion in baboons. Stroke. 1991;22:1276-83.

29. Pham M, Bendszus M. Facing time in ischemic stroke: an alternative hypothesis for collateral failure. Clin Neuroradiol. 2016;26:141-51.

30. Engler RL, Schmid-Schönbein GW, Pavelec RS. Leukocyte capillary plugging in myocardial ischemia and reperfusion in the dog. Am J Pathol. 1983;111:98-111.

Publisher's Note Springer Nature remains neutral with regard to jurisdictional claims in published maps and institutional affiliations. 